\title{
Trattamento chirurgico dell'obesità - adeguamento dell'ordinanza delle prestazioni (Opre)
}

\author{
A. Glättli*
}

* Presidente dello Swiss Study Group for Morbid Obesity

Corrispondenza: Dr. Andreas Glättli Schänzlistrasse 33 CH-3013 Berna

E-mail: glaettli@bauchchirurgie.ch
L'allegato 1 dell'Opre definisce le condizioni per cui, in presenza di un'obesità grave, la presa in carico degli interventi chirurgici, aventi lo scopo di indurre una perdita di peso e di migliorare le comorbidità associate all'obesità, costituisce una prestazione obbligatoria per le casse malattie. Il Swiss Study Group for Morbid Obesity (SMOB) dal momento della sua fondazione nel 1996, si mantiene a disposizione dell'Ufficio federale delle assicurazioni sociali (UFAS) e attualmente dell'Ufficio federale della sanità pubblica (UFSP). In qualità di collaboratore specializzato, lo SMOB gioca un ruolo di consigliere nei confronti della Commissione federale delle prestazioni per l'elaborazione delle raccomandazioni riguardo le prestazioni obbligatorie nell'ambito del trattamento chirurgico de l'obesità.

L'allegato 1 dell'Opre riguardo il trattamento chirurgico dell'obesità, datato 1983, è stato aggiornato nell'ambito di questa collaborazione, e la sua nuova versione è entrata in vigore il $1^{\circ}$ gennaio 2000. Essa è basata essenzialmente sulle conclusioni della «Conferenza di consenso sul trattamento chirurgico dell'obesità in Svizzera» [1], alla quale il PD Dottor F. Horber ha partecipato attivamente in qualità di presidente dello SMOB. Nell'insieme, questa nuova versione dell'allegato 1 riprende i criteri riconosciuti in ambito internazionale per il trattamento chirurgico dell'obesità, ad eccezione dell'indice di massa corporea (Body Mass Index = BMI), il cui limite è stato definito uniformemente a $40 \mathrm{~kg} / \mathrm{m}^{2}$. Nel resto del mondo, il limite è ugualmente fissato a $40 \mathrm{~kg} / \mathrm{m}^{2}$, ma l'indicazione operatoria è ammessa anche se il BMI è $\geq 35 \mathrm{~kg} / \mathrm{m}^{2}$ in presenza di comorbidità severe dovute all'obesità.

Per delle ragioni rimaste finora inspiegabili, il termine «ininterrotto» è stato introdotto nel capoverso d) dell'allegato dell'Opre (tab. 1). Questo voleva dire che la presa in carico da parte delle assicurazioni malattie era obbligatoria solo se «un trattamento adeguato ininterrotto per due anni con lo scopo di indurre una perdita di peso si sia dimostrato inefficace». In maniera evidente per i comuni mortali come per gli specialisti dell'obesità una dieta adeguata della durata di due anni non è realizzabile. Questa modificazione dell'allegato avrebbe dunque potuto significare che, durante i due anni seguenti la sua introduzione, nessun intervento chirurgico per l'obesità sarebbe stato possibile. Difatto, questo piccolo termine ha avuto come conseguenza il rifiuto della presa in carico di un intervento medicalmente indicato in numerosi pazienti.

Questo cambiamento ha motivato diverse reazioni. La Fondazione Svizzera dell'Obesità (FOSO), attraverso il suo presidente Signor von Grünigen, ha protestato per iscritto presso l'UFAS. Anche lo SMOB ha richiesto all'UFSP che il termine «ininterrotto» fosse eliminato dal capoverso d). Inoltre, lo SMOB ha proposto delle definizioni che permettano una interpretazione corretta dei termini «appropriato» e «inefficace». Il gruppo di sostegno dei pazienti di Berna, per mano della Signora Emma Wüthrich, ha lanciato una raccolta di firme per sostenere questa domanda. Altre manifestazioni di sostegno hanno compreso diversi articoli sui giornali ed una trasmissione televisiva su un'emittente locale.

Nel corso dei nostri ripetuti interventi, si è rapidamente rivelato che gli interessi dello SMOB, che rappresenta i pazienti e gli specialisti del trattamento dell'obesità, e quelli della Commissione federale delle prestazioni, la cui più grande preoccupazione è il controllo dei costi della salute, non erano gli stessi. Eppure dei recenti studi hanno confermato che la chirurgia è largamente superiore alle terapie di tipo conservatore nel trattamento dell'obesità grave [4]. Noi consideriamo quindi come un grande successo il fatto di avere ottenuto una soluzione soddisfacente, e questo innanzitutto per i nostri pazienti.

Con le diverse parti coinvolte, lo SMOB esprime la sua soddisfazione nel constatare che, nell'ultima versione dell'allegato 1 dell'Opre, valido a partire dal $1^{\circ}$ gennaio 2005 , il termine «ininterrotto» è stato soppresso dal capoverso d). Questo contribuirà ad una diminuzione della discriminazione alla quale i pazienti con un'obesità grave sono sottoposti quotidianamente.

Lo SMOB redige dal $1^{\circ}$ gennaio 2001 un registro di valutazione secondo il capoverso g) dell'allegato. Quest'ultimo raccoglie tutti gli in- 
Tabella 1

Condizioni dell'allegato 1 dell'Opre valide a partire dal $1^{\circ}$ gennaio 2005 .

\begin{tabular}{|c|c|c|c|}
\hline Provvedimento & $\begin{array}{l}\text { Remunerazione } \\
\text { obbligatoria }\end{array}$ & Condizioni & Decisione valida da \\
\hline \multirow{7}{*}{$\begin{array}{l}\text { Terapia chirurgica dell'obesità (Gastric } \\
\text { Roux-Y Bypass, Gastric Banding, Vertical } \\
\text { Banded Gastroplasty) }\end{array}$} & \multirow[t]{7}{*}{ Sì } & a. Previo colloquio con il medico di fiducia. & \multirow[t]{7}{*}{$1.1 .2000 / 1.1 .2004 / 1.1 .2005$} \\
\hline & & b. Il paziente non deve avere più di 60 anni. & \\
\hline & & c. Il paziente ha un Body Mass Index (BMI) di più di 40. & \\
\hline & & $\begin{array}{l}\text { d. Una terapia adeguata per ridurre il peso, durata due } \\
\text { anni, non ha avuto successo. }\end{array}$ & \\
\hline & & $\begin{array}{l}\text { e. Il paziente soffre di una delle affezioni seguenti: } \\
\text { ipertensione arteriale misurata con un bracciale a } \\
\text { pressione largo; diabete mellito; sindrome d'apnea } \\
\text { del sonno; dislipidemia; affezioni degenerative } \\
\text { invalidanti dell'apparato locomotore; coronaropatie; } \\
\text { sterilità con iperandrogenismo; ovaie policistiche } \\
\text { della donna in età feconda. }\end{array}$ & \\
\hline & & $\begin{array}{l}\text { f. L'operazione va eseguita in un centro ospedaliero } \\
\text { che disponga di una équipe interdisciplinare e con } \\
\text { la necessaria esperienza (chirurgia, psicoterapia, } \\
\text { consulenza nutrizionale, medicina interna). }\end{array}$ & \\
\hline & & $\begin{array}{l}\text { g. Piano di valutazione unitario con statistica delle } \\
\text { quantità e dei costi. }\end{array}$ & \\
\hline
\end{tabular}

terventi effettuati in Svizzera nell'ambito del trattamento chirurgico dell'obesità. Lo SMOB analizza questi dati e li trasmette in forma anonima all'UFSP. Dal momento della creazione di questo registro, nessun centro è autorizzato a praticare la chirurgia dell'obesità se non partecipa alla realizzazione del registro attraverso la trasmissione dei suoi dati. Per evitare qualsiasi incomprensione, lo SMOB ha fatto delle proposizioni concrete per l'interpretazione del capoverso d):

Riconosciuto come trattamento adeguato:

1. consigli nutrizionali dati dai medici (compresi i generalisti) o da un/a dietista diplomato/a e/o

2. trattamento comportamentale compresa la psicoterapia e/o

3. dieta ipocalorica e/o

4. programma di attività fisica e/o

5. trattamento farmacologico;

6. un trattamento appropriato può anche essere effettuato indipendentemente dalla copertura dell'assicurazione malattie (per esempio pagato direttamente dal paziente).

Trattamento inefficace: un trattamento dimagrante appropriato la cui durata totale (cumulabile) è di almeno due anni è considerata come inefficace se, 24 mesi dopo il suo inizio, una perdita di peso minima del $16 \%$ non è stata ottenuta o mantenuta [5]. Questi due anni di trattamento conservativo si possono dividere in differenti programmi appropriati che rispondano ai criteri sopracitati. Per essere preso in conto, ogni singolo trattamento deve durare minimo un mese.

L'obesità, e con essa le comorbidità che le sono associate, costituiranno in un futuro prossimo un importante problema economico e di politica della salute pubblica in Svizzera [6]. L'approccio di questa problematica si può fare solo nell'ambito di una visione interdisciplinare. Il Swiss Study Group for Morbid Obesity continuerà a mantenersi a disposizione dei differenti partner in uno spirito di dialogo costruttivo.

\section{Riferimenti}

1 Golay A, Burckhardt P, Bürgi U, de Marco D, Héraïef E, Horber F, Keller U, Laurent-Jaccard A, Noseda G, Schutz Y, Suter PM. Consensus sur le traitement de l'obésité en Suisse 1999. Schweiz Med Wochenschr 1999;129(Suppl 114):5S-36S.

2 NIH conference. Gastrointestinal surgery for severe obesity. Consensus Development Conference Panel. Ann Intern Med 1991;115(12):956-61.

3 Sauerland S, Angrisani L, Belachew M, Chevallier JM, Favretti F, Finer N, et al. Obesity surgery: evidencebased guidelines of the European Association for Endoscopic Surgery (EAES). Surg Endosc 2004; 19(2):200-21.

4 Sjostrom L, Lindroos AK, Peltonen M, Torgerson J, Bouchard C, Carlsson B, et al. Lifestyle, diabetes, and cardiovascular risk factors 10 years after bariatric surgery. N Engl J Med 2004;351:2683-93

5 Sjostrom CD, Peltonen M, Wedel H, Sjostrom L. Differentiated long-term effects of intentional weight loss on diabetes and hypertension. Hypertension 2000;36(1):20-5

6 sda. BAG-Studie: Übergewicht verursacht 2,7 Milliarden Franken Kosten. Boll Med Svizzeri 2004; 85(40):2110. 